Conclusions There is a wide variation in FCP cut-off levels in the initial diagnosis of IBD as well as in follow-up post-treatment. The FCP cut-off levels vary from country to country. The FCP test requires validation of the available test kits and finding of appropriate cut-off levels for different study populations.

\section{IDDF2019-ABS-0130 GLOBAL SMOKING TRENDS IN INFLAMMATORY BOWEL DISEASE: A SYSTEMATIC REVIEW OF INCEPTION COHORTS}

\begin{abstract}
${ }^{1}$ Venice Sze Wai Li*, ${ }^{2}$ Tom Thomas, ${ }^{3}$ Joht Chandan, ${ }^{1}$ Cheuk Yin Lai, 'Whitney Tang, ${ }^{4}$ Neeraj Bhala, ${ }^{5}$ Gilaad Kaplan, ${ }^{1}$ Siew Chien Ng, ${ }^{6}$ Subrata Ghosh. ${ }^{1}$ Department of Medicine and Therapeutics, Institute of Digestive Disease, State Key Laboratory of Digestive Diseases, LKS Institute of Health Science, Chinese University of Hong Kong, Hong Kong; ${ }^{2}$ Translational Gastroenterology Unit, University of Oxford, UK; ${ }^{3}$ Institute of Applied Health Research, University of Birmingham, UK; ${ }^{4}$ University Hospitals Birmingham NHS Foundation Trust, UK; ${ }^{5}$ Departments of Medicine and Community Health Sciences, University of Calgary, Canada; ${ }^{6}$ NIHR Biomedical Research Centre Birmingham, Institute of Translational Medicine, University of Birmingham, UK
\end{abstract}

\subsection{6/gutjnl-2019-IDDFabstracts. 161}

Background The effect of smoking on inflammatory bowel diseases (IBD) may be heterogeneous across ethnicity and geography. This is a systematic review of smoking prevalence across global IBD cohorts.

Methods A systematic literature search was conducted on Medline and Embase from January 1st, 1946 to April 5th, 2018 to identify population-based studies assessing the prevalence of smoking at diagnosis in inception cohorts of Crohn's disease (CD) or ulcerative colitis (UC). Studies that did not report smoking data from the time of diagnosis or the year of IBD diagnosis were excluded. Prevalence of smoking in IBD was stratified by geography and across time.

Results We identified 56 studies that were eligible for inclusion. Never-smokers in the newly diagnosed CD population in the West has increased over the last two decades, especially in the United Kingdom and Sweden; $+26.6 \%$ and $+11.2 \%$ respectively. Never-smokers at CD diagnosis in newly industrialised nations have decreased over the 1990s and 2000s; China $(-19.36 \%)$. Never-smokers at UC diagnosis also decreased in China; $-15.4 \%$. The former-smoker population at UC diagnosis in China is expanding; 11\%(1990-2006) to 34\%(2011-2013).

Conclusions There has been a reduction in the prevalence of smoking in the IBD cohort in the West. This is not consistent globally. Although smoking prevalence has decreased in the general population of newly industrialised nations, this remains an important risk factor with longer-term outcomes awaiting translation in both UC and CD.

\section{IDDF2019-ABS-0139 EVALUATION OF NARROW BAND IMAGING FINDINGS IN MICROSCOPIC COLITIS AND TARGETED BIOPSIES}

Balaji Bellam*, Siddharth Shukla, Rakseh Kochhar, Anupam Kumar Singh, Satyavathi Rana, Kaushal K Prasad, Narender Dhaka, Sarthak Malik, Neha Berry, Megha Sharma, Saroj Kant Sinha. Postgraduate Institute of Medical Education and Research, Chandigarh, India

10.1136/gutjnl-2019-IDDFabstracts.162
Background Microscopic colitis (MC) is the cause of chronic diarrhoea which is often missed. Histopathology is a gold standard for diagnosis but colonic biopsy has a variable yield in view of normal endoscopy. We evaluated ileocolonic mucosa in suspected MC cases with narrow band imaging (NBI)and described its findings with outcome of targeted biopsy

Methods $\underline{53}$ adults with chronic diarrhea suspected to have MC were recruited after excluding malignancy, celiac disease, small intestinal bacterial overgrowth and inflammatory bowel disease. Routine blood tests, necessary imaging, stool analysis were done. Colonoscopy was done in all with ileal intubation if possible. HDWLE (high definition white light examination) and NBI finding were recorded. Routine biopsies on white light and targeted biopsies on NBI were taken and analysed by an expert gastrointestinal histopathologist as per statements of the European Microscopic Colitis Group 2012

Results 43 patients were diagnosed to have MC [mean age 45.83( \pm 15.92$)$, males - 27]. The WLE revealed normal mucosa in all patients. NBI showed type 1 pit pattern and regular vascular pattern in all patients with MC. Mucosal pattern was honey comb type in all. Focal areas of abnormal vascularity with focally obscure pit pattern was noted more frequently in cases than controls $\{81 \%$ vs $12.5 \%$ (p = $0.052)\}$. Histologically 25(58.1\%) had collagenous colitis (CC), $14(32.5 \%)$ had lymphocytic colitis (LC). Four patients $(9.4 \%)$ had a mixed picture. The yield of WLE and NBI targeted biopsies was the same $(p>0.05)$

Conclusions Colonoscopic NBI findings in MC revealed a hitherto unreported and distinct focal areas of abnormal vascularity with focally obscure pit pattern. There was no significant difference in yield of NBI vs WLE biopsies

\section{IDDF2019-ABS-0141 PREDICTORS OF FAILURE OF ENDOSCOPIC HAEMOSTASIS IN BLEEDING PEPTIC ULCERS}

Harivinthan Sellappan*, Mahadevan Deva Tata, Kandasami Palayan, Gurpreet Singh, Ming Lee Ng. Department of Surgery, Hospital Tuanku Ja'afar Seremban, Malaysia; Department of Surgery, International Medical University, Malaysia

\subsection{6/gutjnl-2019-IDDFabstracts. 163}

Background Bleeding peptic ulcers are the most common cause of non-variceal upper gastrointestinal bleed and are most often amenable to dual modality endoscopic haemostasis. However, a small group of patients fail to respond to endoscopic haemostasis and are often associated with significant morbidity and mortality. We explore the clinical and endoscopic features that predict failure of therapeutic endoscopic haemostasis.

Methods Retrospective case-matched study of consecutive patients who have failed therapeutic endoscopy following bleeding peptic ulcer $(\mathrm{N}=29)$ over a five-year period between 2011 and 2016 in Hospital Tuanku Ja'afar Seremban were age and sex-matched with patients who underwent successful therapeutic endoscopy $(\mathrm{N}=29)$. Failure of therapeutic endoscopy is defined as an end-point of either mortality or need for surgical intervention after at least one attempt at endoscopic haemostasis. Clinical, biochemical and endoscopic features between the two groups were analyzed using Pearson's chisquared test.

Results Patients in both groups had similar baseline characteristics. Mean age was 63.9 in the failed endoscopy group and 
61.3 in the successful endoscopy group $(P=0.077)$. Both groups had an equal number of male and female patients $(\mathrm{P}=1.000)$.

Predictors of failure of endoscopy include initial presentation with haematemesis, initial systolic blood pressure of $\leq 90 \mathrm{mmHg}$, initial heart rate of $\geq 95$ per minute, serum urea $\geq 13 \mathrm{mmol} / \mathrm{L}$, Forrest Ia or $\mathrm{Ib}$ ulcer at first endoscopy and Rockall score of $\geq 5$. (table 1 )

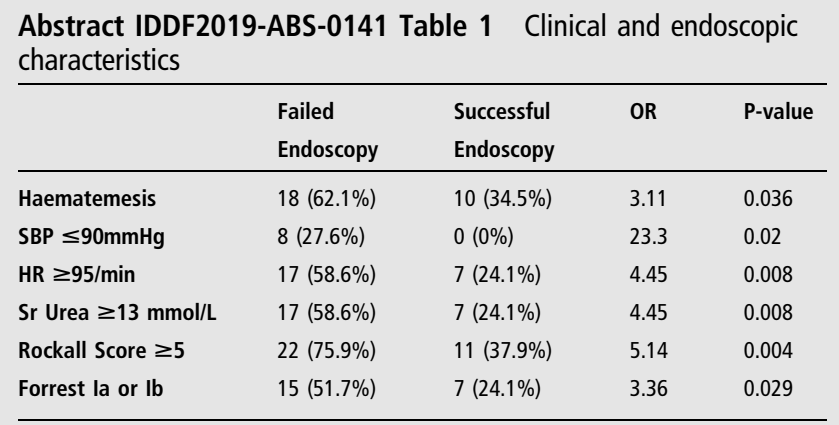

SBP: systolic blood pressure, HR: heart rate, OR: odds ratio

Conclusions It is crucial to identify predictors of failure of endoscopic therapy in bleeding peptic ulcers in order to institute aggressive therapeutic options including early surgical intervention or angioembolization to reduce associated morbidity and mortality.

\section{IDDF2019-ABS-0143 ASSOCIATION BETWEEN BARIATRIC SURGERY AND MACROVASCULAR DISEASE OUTCOMES IN PATIENTS WITH TYPE 2 DIABETES AND SEVERE OBESITY: A META- ANALYSIS OF COHORT STUDIES}

${ }^{1}$ Salman Hussain*, ${ }^{2}$ Ali Nasir Siddiqui, ${ }^{3}$ Abul Kalam Najmi. 'Department of Pharmaceutical Medicine, School of Pharmaceutical Education and Research, Jamia Hamdard, India; ${ }^{2}$ Publication writer, GCE Solutions Inc. Jasola DLF Tower B, New Delhi, India; ${ }^{3}$ Department of Pharmacology, School of Pharmaceutical Education and Research, Jamia Hamdard, India

\subsection{6/gutjnl-2019-IDDFabstracts.164}

Background Severely obese Type 2 Diabetes Mellitus (T2DM) patients are on increased risk of mortality, morbidity and macrovascular complications. Real-world evidence suggested a reduced rate of macrovascular complications following bariatric surgery. So, we undertook this meta-analysis to understand the impact of bariatric surgery in macrovascular disease outcomes in severely obese T2DM patients.

Methods A comprehensive search was performed in PubMed, and Embase database from inception to October 2018. The inclusion criteria were as follows: (a) obese T2DM patients (BMI $>35 \mathrm{~kg} . / \mathrm{m}^{2}$ ) who underwent bariatric surgery (b) provided hazard ratio (HR) or relative risk (RR). Study quality was assessed using the Newcastle-Ottawa Scale. The primary outcome was to assess the impact of bariatric surgery and macrovascular complications risk. Statistical analysis was performed using Review Manager software.

Results This meta-analysis comprised of five studies with a total of 49211 participants (75\% female) of which 14434 underwent bariatric surgery and 34777 underwent usual care. The participants in the bariatric surgery group had a mean age of $48 \pm 8.98$ years, mean BMI of $44.67 \pm 6.3 \mathrm{~kg} / \mathrm{m}^{2}$ and mean diabetes duration and a follow-up period of $5.48 \pm$ 5.11 years and 10.96 years, respectively. Included studies were of high quality.

Participants who underwent bariatric surgery group had significantly lower risk of macrovascular complications as compared to participants who underwent nonsurgery group with a RR of 0.50 (95\% CI: $0.35-0.73$ ), $p=0.0003$ ) (figure 1). Subgroup analysis revealed studies conducted in US showed higher reduction $[\mathrm{RR} \quad 0.41 \quad(95 \%$ CI: $0.32-0.53, \mathrm{p}=$ $<0.00001)]$ in incident macrovascular complications as compared to those conducted in other parts of the world [RR 0.71 (95\% CI: $0.56-0.89), p=0.003]$. The risk of all-cause mortality was also significantly lower in bariatric surgery group (RR of 0.39 [95\% CI: $0.30-0.50$ ], p $=<0.00001$ ).

Conclusions Our meta-analysis supports the benefit of bariatric surgery in reducing macrovascular complications in morbidly obese T2DM patients. However, the observational design of included studies might have precluded the inference despite adjustment of confounding factors. Hence, these findings need to be confirmed in well-designed randomized trials.

\section{IDDF2019-ABS-0144 EFFICACY OF 7-DAY VONOPRAZAN AND AMOXICILLIN DUAL THERAPY AS FIRST- LINE HELICOBACTER PYLORI TREATMENT: PROTOCOL OF MULTI-CENTER, NON- INFERIORITY, RANDOMIZED CONTROL TRIAL}

${ }^{1}$ Sho Suzuki*, 'Takuji Gotoda, ${ }^{2}$ Hitoshi Shibuya, ${ }^{1}$ Chika Kusano, ${ }^{1}$ Hisatomo Ikehara, ${ }^{1}$ Ryoji Ichijima, ${ }^{3}$ Koichi Kawabe, ${ }^{3}$ Toyotaka Kasai, ${ }^{3}$ Hiroyuki Eto, ${ }^{4}$ Yoshioki Yoda, ${ }^{4}$ Masahiko Ohtaka, ${ }^{5}$ Motoki Ohyauchi, ${ }^{5}$ Hirotaka Ito, ${ }^{6}$ Masashi Kawamura, ${ }^{6}$ Yohei Ogata, ${ }^{7}$ Moriyasu Nakahara. ${ }^{1}$ Nihon University School of Medicine, Japan; ${ }^{2}$ Yuri Kumiai General Hospital, Japan; ${ }^{3}$ Fukaya Red Cross Hospital, Japan; ${ }^{4}$ Yamanashi Koseiren Health Care Center, Japan; ${ }^{5}$ Osaki Citizen Hospital, Japan; ${ }^{6}$ Sendai City Hospital, Japan; ${ }^{7}$ Chichibu Municipal Hospital, Japan

\subsection{6/gutjnl-2019-IDDFabstracts. 165}

Background Recent international guidelines recommend fourdrug combination therapies as first-line treatment to overcome Helicobacter pylori antibiotic resistance and achieve sufficient

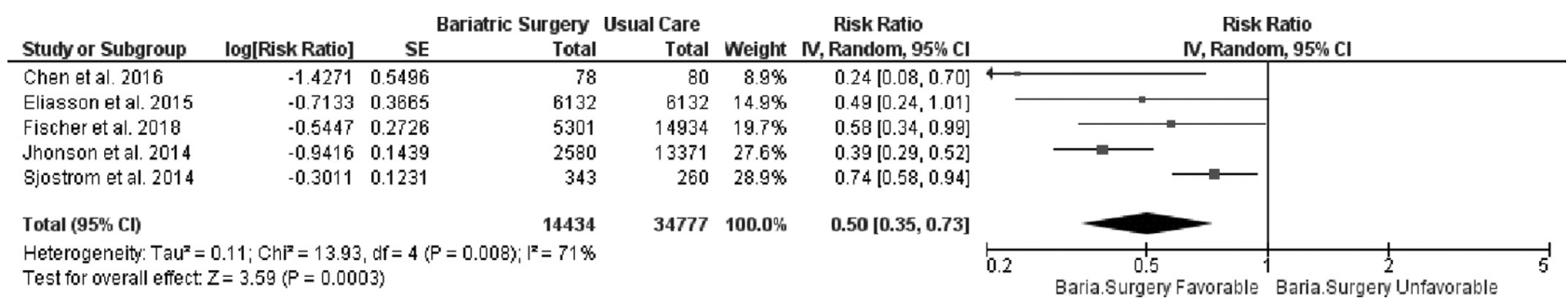

Abstract IDDF2019-ABS-0143 Figure 1 Forest plot showing impact of bariatric surgery in reducing macrovascular complications 